\title{
Formação e atuação de nutricionistas dos Núcleos de Apoio à Saúde da Família
}

\author{
Education and work of dieticians from \\ Family Health Support Centers
}

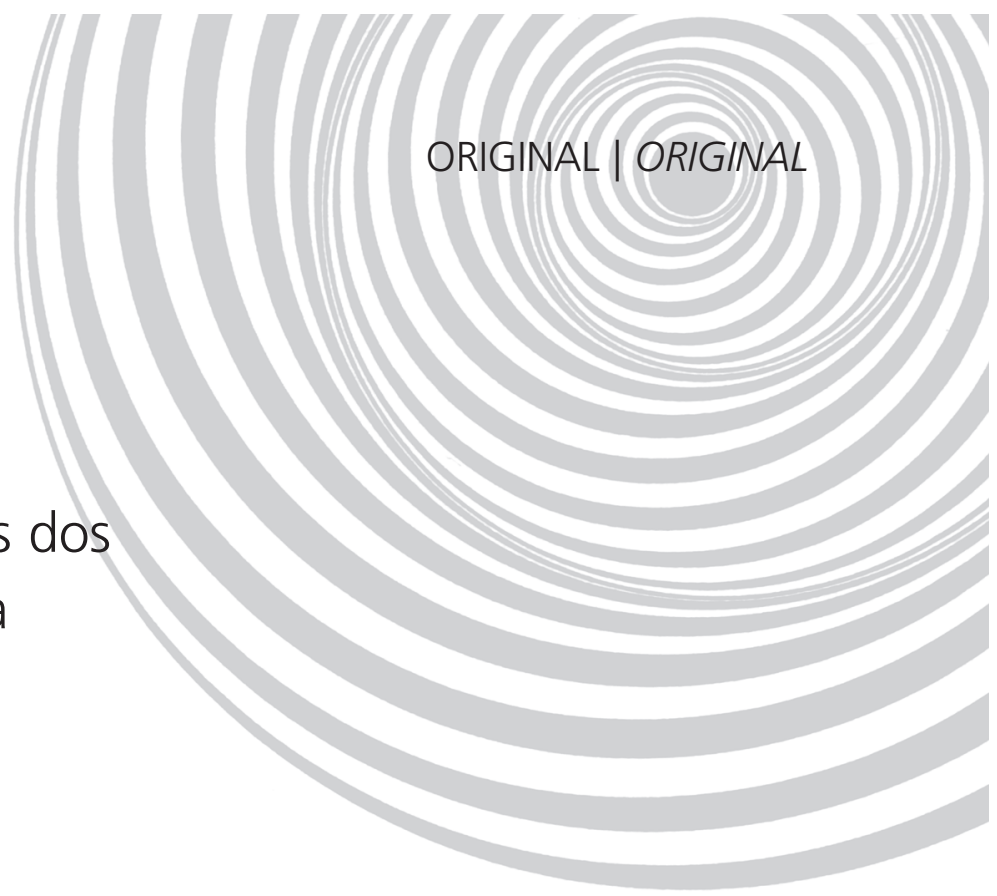

Camilla Botêga AGUIAR ${ }^{1}$

Nilce Maria da Silva Campos COSTA2

RE S U M O

\section{Objetivo}

O presente trabalho tem como objetivo analisar a formação acadêmica e a atuação profissional de nutricionistas dos Núcleos de Apoio à Saúde da Família de Goiás.

\section{Métodos}

Trata-se de estudo descritivo e exploratório, de corte transversal, realizado com nutricionistas atuantes naqueles Núcleos. Para coleta de dados foi utilizado um questionário autoaplicável, com questões abertas e fechadas, elaboradas para caracterizar os nutricionistas, sua formação acadêmica e atuação profissional.

\section{Resultados}

Participaram da pesquisa 22 nutricionistas do sexo feminino, representando 88,0\% do total. Destas, 59,1\% possuíam menos de três anos de formadas; 13,6\% fizeram especialização em Saúde da Família e apenas 27,3\% sentiam-se capacitadas para a atuação profissional mediante reflexão sobre a realidade socioeconômica, política e cultural do território. Em relação à atuação, 80,0\% foram contratadas em caráter temporário, o que pode comprometer o vínculo entre o profissional e a população. Ainda, 36,4\% atuavam há menos de 1 ano; 18,2\% foram capacitadas antes do início das atividades; e 59,1\% sentiam-se pouco capacitadas para trabalhar nos núcleos. Apenas 9,1\% das participantes discutiam sobre matriciamento nas reuniões e utilizavam referências sobre a ferramenta. O trabalho em equipe foi apontado como facilitador das ações realizadas, e o não conhecimento do papel dos Núcleos como um fator que dificulta.

\section{Conclusão}

Os nutricionistas dos Núcleos possuem pouca experiência profissional, sentem-se pouco qualificados para atuar em saúde da família e apresentam dificuldades de compreensão da realidade social, pois a formação acadêmica não proporcionou segurança para atuação na área, assim como é precário o conhecimento sobre os Núcleos.

Palavras-chave: Atenção Básica à Saúde. Nutricionista. Recursos Humanos.

1 Instituto Federal de Goiás, Faculdade de Nutrição. Campus Goiânia, R. 227, Quadra 68, s/n., Setor Leste Universitário, 74605-080, Goiânia, GO, Brasil. Correspondência para/Correspondence to: NMSC COSTA. E-mail: <nilcecosta58@gmail.com>. 2 Universidade Federal de Goiás, Faculdade de Nutrição. Goiás, GO, Brasil.

Artigo elaborado a partir da dissertação de CB AGUIAR, intitulada: "Formação acadêmica e atuação profissional de nutricionistas dos Núcleos de Apoio à Saúde da Família de Goiás". Universidade Federal de Goiás; 2013. 


\section{A B S T R A C T}

\section{Objective}

The aim of this study was to analyze the education and work of dieticians from the Family Health Support Centers in the state of Goiás.

\section{Method}

This is a descriptive, exploratory, cross-sectional study with dieticians from these centers. The dieticians answered a self-administered questionnaire with open and closed questions that characterized them, their education, and their work.

\section{Research}

Twenty-two female dieticians from Family Health Support Centers participated in the study, representing $88.0 \%$ of the total. Of these, $59.1 \%$ had graduated less than three years ago; $13.6 \%$ specialized in family health; and only $27.3 \%$ felt capable of performing their job based on a reflection about the socioeconomic, political, and cultural reality of the territory. Regarding work, $80 \%$ had temporary job contracts, which can compromise the bond between the professionals and the population; 36.4\% had been working for less than one year; $18.2 \%$ received training before they started working; and $59.1 \%$ did not feel sufficiently trained to work in the centers. Only $9.1 \%$ of the participants discussed matriciamento (support given by a multidisciplinary team to another multidisciplinary team) in the meetings and used tool-related references. Team work was considered a facilitator of their actions, and not knowing the role of Family Health Support Centers was considered a hindrance.

\section{Conclusion}

Dieticians from the Family Health Support Centers in the state of Goiás have little professional experience, do not feel sufficiently trained to work in family health, and have difficulties understanding the local reality, as education did not give them confidence to work in the area and they have little knowledge about the centers.

Keywords: Primary Health Care. Nutritionist. Human Resources.

\section{N T R O D U ÇÃ O}

O nutricionista é um profissional do setor de saúde, que tem como objeto de trabalho a alimentação do homem, no plano individual e coletivo' ${ }^{1}$. A formação do nutricionista vem sendo discutida na América Latina desde a década de 1960, com o objetivo de definir o perfil profissional, aprimorar a formação acadêmica e adequá-la ao mercado de trabalho, resultando em recomendações para a organização dos currículos dos cursos de Nutrição².

Os movimentos de mudança na formação têm-se articulado de forma progressiva com as estratégias de reorganização das práticas de saúde, o que tem proporcionado a construção de novos paradigmas de educação e saúde, expressos em modelos pedagógicos e assistenciais que objetivam se aproximar das necessidades da população ${ }^{3}$.
As orientações curriculares e pedagógicas para a formação do profissional de nutrição têm recebido a influência das transformações ocorridas no ensino superior no Brasil ${ }^{4}$, como as proporcionadas pelas Diretrizes Curriculares Nacionais $(D C N)^{5}$ e pelo Programa de Reorientação da Formação Profissional em Saúde (Prosaúde) ${ }^{6}$.

Em 2001, o Ministério da Educação instituiu as DCN para os cursos de graduação em Nutrição, com ênfase no desenvolvimento de competências e habilidades profissionais, que desafiam as universidades a transformar currículos e processos de ensino-aprendizagem-avaliação. Tais diretrizes direcionam a uma estruturação qualitativa dos cursos, voltada às necessidades sociais da saúde da população, com ênfase no Sistema Único de Saúde (SUS) 7 .

O Sistema Único de Saúde é, preferencialmente, a primeira forma de atendimento à população ${ }^{8}$. Desde 1994, o Brasil experimenta a 
implementação da Estratégia da Saúde da Família (ESF), orientada por uma ação multidisciplinar voltada para a promoção da saúde do indivíduo, da família e da comunidade, e consubstanciada na Saúde da Família9 . A realização plena dos objetivos dessa estratégia guarda estreita dependência com a capacidade de desenvolver e implementar ações na área de Alimentação e Nutrição, que avancem na conquista da segurança alimentar e nutricional dos brasileiros ${ }^{10}$.

A participação ativa do nutricionista na ESF, porém, ainda não está regulamentada, muito embora tenha aumentado sua inserção na assistência à saúde da população, em razão da implantação dos Núcleos de Apoio à Saúde da Família (Nasf) nas diferentes regiões do País?

Os Núcleos de Apoio à Saúde da Família vieram contribuir com as equipes de ESF, tendo como preceito básico um trabalho interdisciplinar e de matriciamento. Os Nasf atuam de acordo com as peculiaridades regionais e têm o objetivo de promover saúde de forma mais eficiente, contemplando profissões e áreas inexistentes nas equipes de ESF. O trabalho desenvolvido entre ESF e Nasf deve ser organizado sob o eixo do trabalho coletivo e da gestão participativa, com a criação de espaços coletivos de discussão e planejamento conjunto ${ }^{11}$.

O presente trabalho teve como objetivo analisar a formação acadêmica e a atuação profissional de nutricionistas dos Nasf do estado de Goiás.

\section{MÉ T O D O S}

Foi realizado um estudo descritivo e exploratório, de corte transversal, com nutricionistas dos Nasf do estado de Goiás. Como instrumento de coleta de dados foi utilizado um questionário autoaplicável, visando conhecer as questões que interessavam ser investigadas.

O questionário é um instrumento de coleta de dados caracterizado por possuir questões fechadas e/ou abertas, articuladas entre si, com vistas ao levantamento de informações escritas pelos sujeitos pesquisados e que sejam pertinentes ao objeto de investigação ${ }^{12}$. Na verdade, o questionário "supõe uma seleção operada pelo pesquisador, cobrindo os temas abordados" 13 .

O questionário foi construído com questões fechadas e abertas. Nas primeiras, poderia ser assinalada mais de uma opção por pergunta. Em todas as questões, havia um espaço para redação de justificativas, caso o respondente julgasse necessário.

O questionário foi elaborado a partir de três categorias de análise: 1) caracterização dos participantes em relação a idade, sexo, tempo de formação e experiência profissional; 2) formação acadêmica do nutricionista, com questões elaboradas com base nas DCN para os cursos de Nutrição5; e 3) atuação profissional do nutricionista nos Nasf, com questões adaptadas do instrumento do Observatório de Políticas em Segurança Alimentar e Nutricional (OPSAN) $)^{14}$.

Os dados foram coletados em dezembro de 2012 e submetidos a análise descritiva, com cálculo das frequências absolutas e relativas.

O estudo foi aprovado pelo Comitê de Ética em Pesquisa da Universidade Federal de Goiás (UFG), sob o Parecer nº 246.578/2012.

\section{RESULTADOS}

Participaram da pesquisa 22 (88.0\%) nutricionistas dos Nasf de Goiás, do sexo feminino, com predominância de idade entre 20 e 40 anos $(95,4 \%)$ e experiência profissional menor que 3 anos (50.0\%).

Quinze $(68,2 \%)$ nutricionistas possuíam especialização em andamento ou concluída, e destas, apenas $13,6 \%$ a fizeram na área de Saúde da Família. Além disso, 9,1\% cursavam mestrado profissional em ensino na saúde.

A maioria $(81,8 \%)$ delas ingressou nos Nasf via contratação temporária, com carga ho- 
rária semanal de 40 horas. Destas, oito $(36,4 \%)$ participantes afirmaram trabalhar também em outros locais e áreas, como academias e consultórios.

\section{Formação acadêmica dos nutricionistas}

Dezoito nutricionistas $(81,8 \%)$ pesquisadas consideraram ter recebido uma formação acadêmica generalista, humanista, crítica e reflexiva. Destes, 40,9\% demonstraram possuir uma percepção ampliada do conceito de formação para além da graduação: "Entendo formação: graduação, especialização, mestrado, educação permanente e qualificação profissional. Essa formação certamente não obtive na academia, mas na prática e na qualificação profissional" (Participante 6).

Por outro lado, apenas 27,3\% das participantes da pesquisa reconheceram-se aptas a refletir sobre a realidade econômica, política, social e cultural do território de atuação.
As Diretrizes Curriculares Nacionais preconizam como competências gerais dos profissionais de saúde. Neste estudo, observou-se que assumir posições de liderança foi referido por $31,8 \%$ nutricionistas e, destes, 27,3\% sentiam-se aptos a exercer a gestão e liderança na equipe de saúde. Em relação à Atenção à Saúde, os participantes afirmaram possuir aptidão para desenvolver ações de promoção (68,7\%), prevenção e reabilitação $(45,4 \%)$ da saúde, tanto em nível individual quanto coletivo.

Quatro (18,2\%) nutricionistas ressaltaram ter desenvolvido, durante a graduação, competências de comunicação verbal e não verbal, habilidades de escrita e de leitura, domínio de uma língua estrangeira e de tecnologias de comunicação/informação.

As habilidades e competências específicas para atuação na Atenção Básica à Saúde, adquiridas durante a formação acadêmica, estão apresentadas na Tabela 1.

Tabela 1. Habilidades e competências específicas de nutricionistas, para atuação na Atenção Básica, adquiridas na formação, na visão de nutricionistas dos Núcleos de Apoio à Saúde da Família, Goiás, 2012.

\begin{tabular}{|c|c|c|}
\hline \multirow{2}{*}{ Dados } & \multicolumn{2}{|c|}{ Respostas } \\
\hline & $\mathrm{n}$ & $\%$ \\
\hline $\begin{array}{l}\text { Aplicar os conhecimentos sobre composição, propriedades e transformações dos alimentos e seu aproveitamento } \\
\text { pelo organismo humano, na atenção dietética. }\end{array}$ & 19 & 86,4 \\
\hline Contribuir para promover, manter e/ou recuperar o estado nutricional de indivíduos e grupos populacionais. & 18 & 81,8 \\
\hline Investigar e aplicar conhecimentos com visão holística do ser humano, integrando equipes multiprofissionais. & 14 & 63,6 \\
\hline Atuar em equipes multiprofissionais de saúde e de terapia nutricional. & 13 & 59,1 \\
\hline $\begin{array}{l}\text { Realizar diagnósticos e intervenções na área de alimentação e nutrição, considerando a influência sociocultural e } \\
\text { econômica que determina a disponibilidade, consumo e utilização biológica dos alimentos pelo indivíduo e pela } \\
\text { população. }\end{array}$ & 13 & 59,1 \\
\hline $\begin{array}{l}\text { Atuar em equipes multiprofissionais destinadas a planejar, coordenar, supervisionar, implementar, executar e avaliar } \\
\text { atividades na área de alimentação e nutrição e de saúde. }\end{array}$ & 13 & 59,1 \\
\hline $\begin{array}{l}\text { Atuar em políticas e programas de educação, segurança e vigilância nutricional, alimentar e sanitária, visando à } \\
\text { promoção da saúde em âmbito local, regional e nacional. }\end{array}$ & 10 & 45,4 \\
\hline $\begin{array}{l}\text { Reconhecer a saúde como direito e atuar de forma a garantir a integralidade da assistência, entendida como conjun- } \\
\text { to articulado e contínuo das ações e serviços preventivos e curativos, individuais e coletivos, exigidos para cada caso, } \\
\text { em todos os níveis de complexidade do sistema. }\end{array}$ & 10 & 45,4 \\
\hline $\begin{array}{l}\text { Atuar na formulação e execução de programas de educação nutricional e de vigilância nutricional, alimentar e } \\
\text { sanitária. }\end{array}$ & 9 & 40,9 \\
\hline $\begin{array}{l}\text { Avaliar, diagnosticar e acompanhar o estado nutricional; planejar, prescrever, analisar, supervisionar e avaliar dietas e } \\
\text { suplementos dietéticos para indivíduos sadios e enfermos. }\end{array}$ & 5 & 22,7 \\
\hline
\end{tabular}




\section{Atuação profissional nos Nasf}

A maioria $(59,1 \%)$ das nutricionistas considerou-se pouco capacitada para exercer as atividades sob sua responsabilidade, demonstrando insegurança para o trabalho: "Ainda necessito de mais capacitação para desenvolver melhor o meu trabalho" (Participante 13).

Cinco $(22,7 \%)$ nutricionistas relataram ter recebido algum tipo de capacitação antes de ingressar nos Nasf: "Ficamos em educação permanente semanal por 8 meses antes de começar no território" (Participante 9). As demais consideraram ter aprendido com a experiência prática diária. De acordo com 12 (54,9\%) nutricionistas, não havia previsão de realização de atividades de educação continuada.

Doze $(54,9 \%)$ participantes apontaram que a prática nos Nasf ocorria de forma integrada e contínua com as demais instâncias de saúde, por meio da intersetorialidade, com reuniões em grupos e referência e contrarreferência. A maioria $(72,7 \%)$, no entanto, não soube descrever como se dá essa integração.

Vinte $(90,9 \%)$ nutricionistas referiram realizar reuniões com as equipes dos Nasf. Os principais pontos debatidos foram o planejamento (90,9\%) e a discussão de casos clínicos (40,9\%). Atividades propostas pelas diretrizes dos Nasf, entretanto, foram abordadas poucas vezes, como por exemplo, o matriciamento, citado por apenas duas participantes $(9,1 \%)$, ferramenta utilizada para oferecer um suporte técnico-pedagógico às ESF.

Sete $(31,8 \%)$ participantes afirmaram discutir o trabalho com a ABS, enquanto $27,3 \%$ destacaram que raramente ocorriam reuniões com a ESF da área de abrangência, e 22,7\% referiram inocorrência de encontros periódicos para discussão com os Nasf do município.

O Núcleo de Apoio à Saúde da Família organiza seu processo de trabalho com foco nos territórios de sua responsabilidade, e o monitoramento dessa área de abrangência é importante para a eficácia das ações e para a realização de novos planejamentos e metas ${ }^{15}$. Neste estudo, porém, os territórios nunca eram monitorados ou raramente o eram $(36,4 \%)$.

Mais da metade $(63,6 \%)$ dos nutricionistas relataram realizar uma análise da situação de saúde da população utilizando os dados dos sistemas de informação, como Sistema de Informação da Atenção Básica (SIAB) e Sistema de Vigilância Alimentar e Nutricional (SISVAN), os dados do território, das equipes de ESF e Agentes Comunitários de Saúde e das visitas domiciliares. Os métodos utilizados para realizar o diagnóstico alimentar e nutricional foram a avaliação antropométrica $(77,3 \%)$ e a frequência alimentar $(68,2 \%)$.

Neste estudo, observou-se que as referências mais utilizadas foram o Caderno de Atenção Básica para os Nasf, a Portaria do Nasf, o Guia Alimentar para a População Brasileira, respectivamente destacados por 20 (90,9\%), 19 $(86,4 \%)$ e $18(81,8 \%)$ nutricionistas. Algumas referências, porém, raramente são usadas pelas nutricionistas participantes desta pesquisa, como a Matriz de ações de alimentação e nutrição na ABS e o matriciamento, utilizadas apenas por $9,1 \%$.

Várias ações podem ser desenvolvidas nos Nasf, estabelecidas pelo caderno de diretrizes. Dentre elas, as mais realizadas pelas nutricionistas que participaram desta investigação podem ser visualizadas na Figura 1.

As nutricionistas dos Nasf de Goiás realizavam o monitoramento dos programas e ações de alimentação e nutrição que compõem a agenda programática da Atenção Primária à Saúde, como Bolsa Família, SISVAN, Programa Nacional de Suplementação de Ferro (PNSF) e Vitamina A.

As ações de promoção de Práticas Alimentares Adequadas e Saudáveis (PAAS) ocorriam para $81,8 \%$ por meio de palestras, grupos, oficinas, orientações individuais, capacitações e rodas de conversa. Diversas ações eram executa- 




Figura 1. Ações desenvolvidas por nutricionistas, Núcleos de Apoio à Saúde da Família. Goiás. 2012.

das em parceria com outros profissionais por 90,9\%, como promoção do aleitamento materno e prevenção e tratamento de doenças crônicas não transmissíveis. A promoção de Segurança Alimentar e Nutricional (SAN) era realizada principalmente nas ações de PAAS $(86,4 \%)$ e atendimentos individuais $(22,7 \%)$.

Todas as nutricionistas destacaram não ter elaborado protocolo de atenção nutricional individual ou coletivo, e sim, desenvolvido Projetos Terapêuticos Singulares (PTS), especialmente nas doenças e agravos não transmissíveis $(59,1 \%)$ e de acordo com as demandas do Nasf $(22,7 \%)$.

As pesquisadas ressaltaram como melhoria nas condições de saúde, após implantação do Nasf: mudanças no estilo de vida (40,9\%), mudanças de hábitos alimentares (36,4\%) e melhoria na atenção e assistência à Saúde (27,3\%). Duas nutricionistas citaram melhoria no acolhimento, na relação das equipes com a população, na informação e na qualidade de vida da população.

As nutricionistas consideraram o trabalho em equipe (50,0\%) e acolhimento das $\operatorname{ESF}(22,7 \%)$ como fatores facilitadores das ações nos Nasf. Já em relação a fatores dificultadores, foi citada a falta de recursos materiais e de infraestrutura $(59,1 \%)$ e a falta de conhecimento sobre o papel dos Nasf (36,4\%).

\section{DIS C U S S Ã O}

A caracterização das nutricionistas pesquisadas revela semelhança com os encontrados no estudo de Santana \& Ruiz-Moreno ${ }^{16}$, que também encontraram preponderância do sexo feminino e faixa etária entre 20 e 40 anos (89,5\%).

Apesar da busca por atualização profissional dos participantes deste estudo, poucos a realizaram na área de Saúde da Família, resultado próximo aos achados de Fagundes ${ }^{17}$, que pesquisou nutricionistas dos Nasf de todo o Brasil. A oferta de formação especializada nessa área ainda é insuficiente, e há baixa procura por esses cursos, o que pode estar relacionado ao vínculo precário dos contratos de trabalho temporários dos nutricionistas que atuam nos Nasf. Por outro lado, foi observado que os nutricionistas admitidos nos Nasf por meio de concurso público preocuparam-se em investir em sua qualificação profissional, por meio da realização de cursos de especialização na área. 
Embora as Diretrizes Curriculares Nacionais preconizem a importância da formação generalista, humanista, crítica e reflexiva, apenas $31,8 \%$ das nutricionistas sentiam-se capacitadas a atuar com base em reflexões acerca da realidade econômica, política, social e cultural, o que pode dificultar a atuação profissional. Esse dado representa uma contradição com o perfil profissional levantado pelas participantes, apresentado anteriormente. Como os Nasf estão localizados na maioria em regiões periféricas e mais vulneráveis, é necessário o desenvolvimento de competências para a compreensão da realidade local, a fim de garantir os direitos e a segurança alimentar e nutricional da população8.

A formação acadêmica deve estar vinculada às necessidades sociais de saúde ${ }^{18}$. Faz-se necessário desenvolver o potencial social e valorativo para que se formem profissionais humanistas, éticos e comprometidos com as questões sociais e de cidadania ${ }^{19}$.

Percebe-se grande dificuldade dos participantes em assumir posições de liderança e atuar em gerenciamento e administração na Atenção Básica à Saúde, o que pode ser atribuído às fragilidades no desenvolvimento de um trabalho sistemático e estratégico, bem como à pouca experiência profissional e à alocação inadequada e/ou insuficiente de recursos e materiais.

Destaca-se a importância que as participantes deram aos saberes adquiridos com a experiência na prática profissional, ressaltando um processo de construção contínuo, conforme discutem Banduk et al. ${ }^{20}$. Para Aguiar \& Ribeiro7, o saber que se elabora segundo uma ordem pessoal e a partir da experiência de cada um, é gerado e compartilhado culturalmente e está em constante transformação. A competência é aprimorada, no dia a dia, no processo de ensino-aprendizagem e na vivência do sujeito.

Assim, a reflexão quanto à formação em nível de graduação não deve estar limitada à adequação das disciplinas que constituem o currículo, mas deve ocorrer de forma transversal, com novas metodologias e incentivo a atividades que contribuam para desenvolvimento de habilidades de comunicação, liderança, tomada de decisões, ética e trabalho em equipe, de forma interdisciplinar ${ }^{21}$.

Dentre as formas de admissão aos Nasf, houve a predominância de contratos temporários de trabalho, fato que pode gerar descontinuidade das ações desenvolvidas e levar à desmotivação dos profissionais que convivem com a iminência do fim dos contratos, o que pode comprometer a efetividade da ESF. Em uma pesquisa na Atenção Primária à Saúde de Campinas (SP) 22, o vínculo frágil foi atribuído à alta rotatividade de profissionais, o que enfraquece a continuidade do tratamento e, por conseguinte, compromete sua eficácia.

Nesta pesquisa, observou-se uma população com pouca experiência profissional, o que pode ser fruto do aumento de demanda na área e da alta rotatividade dos profissionais. Segundo o Consenso sobre Habilidades e Competências do Nutricionista no Âmbito da Saúde Coletiva21, o profissional iniciante atua por meio de regras e normas e requer orientação, limitando-se sua atuação à rotina do serviço. Porém, a atuação no Nasf exige profissionais experientes, capazes de desenvolver ações pautadas no trabalho interdisciplinar, intersetorial, ético, resolutivo, longitudinal, acolhedor, com vínculo e responsabilização.

Apesar de $72,7 \%$ das pesquisadas cumprirem 40 horas semanais de trabalho, conforme preconizado pela Portaria que criou os Nasf ${ }^{11}$, $31,8 \%$ exerciam a profissão também em outros locais ou áreas de atuação, ultrapassando a carga horária de trabalho de 8 horas/dia, resultado semelhante ao obtido por Miranda et al. ${ }^{23}$

A falta de capacitação inicial para ingresso nas atividades do Nasf pode prejudicar a execução e eficácia das ações do nutricionista, pelo desconhecimento dos objetivos do Nasf e do papel profissional, bem como pela falta de domínio das competências. Trata-se de uma das dificuldades apontadas para a implementação da ESF como política como modelo assistencial de saúde ${ }^{24}$. É importante ainda refletir sobre a metodologia e 
os conteúdos abordados, que deixaram a desejar na visão das nutricionistas participantes, fato que merece ser repensado pelos organizadores das novas capacitações.

De acordo com 54,4\% das nutricionistas, não havia previsão de atividades de educação continuada. É necessário que os gestores programem treinamentos aos profissionais, sendo este um fundamento do sistema e responsabilidade do próprio governo ${ }^{17}$.

O planejamento, instrumento importante na implementação dos Nasf ${ }^{25}$, foi um ponto relevante levantado nas reuniões realizadas pelas equipes. O matriciamento, porém, diretriz fundamental dos Nasf ${ }^{11}$, foi pouco destacado nas reuniões e no suporte teórico na prática das nutricionistas, o que pode colaborar para a ocorrência de dificuldades na atuação profissional nos Nasf e, consequentemente, no aumento da sensação de incapacidade. Ressalta-se a importância do profissional apoiador matricial que, por meio de suporte técnico pedagógico, contribui com intervenções para aumentar a capacidade de solucionar os problemas de saúde da população ${ }^{26}$.

O trabalho do nutricionista na Atenção Básica à Saúde ainda está principalmente voltado para ações no nível individual e restrito aos limites físicos da unidade de saúde. Esse modo de atuação parece estar intimamente ligado a uma formação flexneriana e fragmentada, que dificulta ações em equipe. Os usuários do SUS têm expectativas da atuação profissional em nível grupal e coletivo, com objetivos educacionais ou de convivência ${ }^{27}$.

Fagundes ${ }^{17}$ destaca que os nutricionistas dos Nasf estão assumindo ações de atenção nutricional individual, porque em muitos locais não havia tal atendimento e existe uma demanda reprimida, com elevado número de pacientes em espera. Esse fato colabora para dificuldades na implementação dos Nasf.

O nutricionista, membro da equipe do Nasf, desenvolve suas ações prioritariamente com a equipe da ESF da respectiva área de abrangên- cia. Dentre as atribuições regulares dos profissionais do Nasf, o atendimento clínico individual deveria ocorrer apenas ocasionalmente. Quando o usuário necessitar de cuidado nutricional específico, este deve ser operacionalizado por meio do PTS ${ }^{15}$.

Nesta pesquisa, percebeu-se que o desenvolvimento dos PTS ainda é pequeno, frente à importância desse instrumento de ação nos Nasf. Conforme a Portaria GM 154 de 2008'2, que instituiu os Nasf, o PTS deve ser construído em parceria com a ESF, que tem a responsabilidade em executá-lo.

O trabalho interdisciplinar e multiprofissional é fundamental para a boa atuação profissional, e é um componente imprescindível para atuação no Nasf, pois pressupõe interação de saberes e experiências entre profissionais da equipe, com foco na integralidade ${ }^{27}$.

Minimizar a carência da estrutura e oferecer condições adequadas para o trabalho em nível local - com a disponibilização de recursos, equipamentos antropométricos, referências e materiais técnicos -, são questões primordiais para manter a motivação dos profissionais e não gerar/potencializar dificuldades em meio a tantos desafios já impostos ${ }^{17}$.

Relatos de experiências positivas de inserção do nutricionista em equipes multidisciplinares trazem à tona, entretanto, a ignorância sobre as funções desse profissional por parte de gestores de diferentes programas de saúde, bem como a insuficiência das orientações nutricionais realizadas por outros profissionais que não sejam da área ${ }^{28,29}$, fatores apontados como dificuldades para a atuação dos Nasf.

\section{O N C L US Ã O}

Os nutricionistas que atuam nos Nasf de Goiás são profissionais com pouca experiência profissional e escassa qualificação para atuação, com dificuldades em refletir sobre os determinantes socioeconômicos, políticos e culturais. 
Além disso, a contratação temporária pode comprometer o vínculo entre os profissionais e a população. Soma-se a isso a pouca utilização de materiais referenciais do Nasf, como o matriciamento, e o pouco desenvolvimento de Projetos Terapêuticos Singulares.

Desafios precisam ser enfrentados para melhorar a formação acadêmica em nutrição, como rever a perspectiva pedagógica tradicional e hegemônica, com a transmissão vertical de conhecimento. Este estudo aponta que a formação do nutricionista irá demandar uma reflexão séria sobre seu papel social e sua inserção nos Nasf.

Os processos de transformação no âmbito de recursos humanos, entretanto, são complexos e também conflituosos, e podem necessitar de um longo tempo de construção.

Assim, há necessidade de mais estudos sobre essa realidade para o aperfeiçoamento da formação acadêmica e da atuação profissional na área, contribuindo para a implementação dos Nasf.

\section{REFERÊ N CIAS}

1. Ypiranga L. Delimitação do objeto de trabalho do nutricionista: subsídios para uma discussão. Saúde Debate. 1990; 29:62-9.

2. Costa NMSC. A formação do nutricionista: educação e contradição. 2ª ed. Goiânia: UFG; 2002.

3. Braccialli LAD, Oliveira MAC. Desafios na formação médica: a contribuição da avaliação. Rev Bras Educ Med. 2012; 36(2):280-8.

4. Soares NT, Aguiar AC. Diretrizes curriculares nacionais para os cursos de nutrição: avanços, lacunas, ambiguidades e perspectivas. Rev Nutr. 2010; 23(5):895-905. http://dx.doi.org/10.1590/S1415-5 2732010000500019

5. Brasil. Ministério da Educação. Conselho Nacional de Educação. Resolução CNE/CES n 5/2001. Institui diretrizes curriculares nacionais do curso de graduação em nutrição. Diário Oficial da União. 2001; 9 nov, p.39, Seção 1.

6. Brasil. Ministério da Saúde. Portaria Interministerial n³.019/2007. Dispõe sobre o Programa Nacional de Reorientação da Formação Profissional em Saúde
- Pró-Saúde - para os cursos de graduação da área da saúde. Brasília: Ministério da Saúde; 2007.

7. Aguiar AC, Ribeiro ECO. Conceito e avaliação de habilidades e competência na educação médica: percepções atuais dos especialistas. Rev Bras Educ Med. 2010; 34(3):371-8.

8. Cervato-Mancuso AM, Tonácio LV, Silva ER, Vieira VL. A atuação do nutricionista na atenção básica à saúde. Ciênc Saúde Colet. 2012; 17(12):3289-300.

9. Brasil. Ministério da Saúde. Portaria n².488/2011. Aprova a Política Nacional de Atenção Básica, estabelecendo a revisão de diretrizes e normas para a organização da Atenção Básica, para a Estratégia Saúde da Família (ESF) e o Programa de Agentes Comunitários de Saúde (PACS). Brasília: Ministério da Saúde; 2011.

10. Assis AMO, Santos SMC, Freitas MCS, Santos JM, Silva MCM. O Programa Saúde da Família: contribuições para uma reflexão sobre a inserção do nutricionista na equipe multidisciplinar. Rev Nutr. 2002; 15(3):255-66. http://dx.doi.org/10.1590/S 1415-52 732002000300001

11. Brasil. Ministério da Saúde. Portaria GM nº 154/2008. Cria os Núcleos de Apoio à Saúde da Família - NASF. Brasília: Ministério da Saúde; 2008.

12. Severino AJ. Metodologia do Trabalho Científico. $23^{a}$ ed. São Paulo: Cortez; 2007.

13. Oliveira MLC. Comitê de ética em pesquisa na Brasil: um estudo das representações sociais. Brasília: Universa; 2004.

14. Brasil. Organização Pan Americana da Saúde. Observatório de Políticas de Segurança Alimentar e Nutrição. Como estão sendo realizadas as ações de Alimentação e Nutrição nos Núcleos de Apoio à Saúde da Família? Texto de Sistematização da Rede Nutri. Brasília: Ministério da Saúde; 2010 [acesso 2012 nov 11]. Disponível em: http://ecos-redenutri. bvs.br

15. Brasil. Ministério da Saúde. Secretaria de Atenção à Saúde. Diretrizes do NASF: Núcleos de Apoio à Saúde da Família. Brasília: Ministério da Saúde; 2010. Série Cadernos de Atenção Básica, nº 27.

16. Santana TCM, Ruiz-Moreno L. Formação do nutricionista atuante no Programa Nacional de Alimentação Escolar. Nutrire. 2012; 37(2):183-98.

17. Fagundes AA. A atuação do nutricionista nos Núcleos de Apoio à Saúde da Família [doutorado]. Brasília: Universidade de Brasília; 2013.

18. Costa GD, Cotta RMM, Ferreira MLSM, Reis JB, Reis F, Castro SC. Saúde da família: desafios no processo de reorientação do modelo assistencial. Rev Bras Enferm. 2009; 62(1):113-8. 
19. Recine E, Gomes RCF, Fagundes AA, Pinheiro ARO, Teixeira BA, Sousa JS, et al. A formação em saúde pública nos cursos de graduação de nutrição no Brasil. Rev Nutr. 2012; 25(1):21-33. http://dx.doi. org/10.1590/S1415-52732012000100003

20. Banduk MLS, Ruiz-Moreno L, Batista NA. A construção da identidade profissional na graduação do nutricionista. Interface. 2009; 13(28):111-20.

21. Recine E, Mortoza AS. Consenso sobre habilidades e competências do nutricionista no âmbito da saúde coletiva. Brasília: Observatório de Políticas de Segurança e Nutrição; 2013.

22. Campos RO, Gama CA, Ferrer AL, Santos DVD, Stefanello S, Trapé TL, et al. Saúde mental na atenção primária à saúde: estudo avaliativo em uma grande cidade brasileira. Ciênc Saúde Colet. 2011; 16(12):4643-52.

23. Miranda DEGA, Pereira CHC, Paschoini TB, Quaglio T. O perfil de atuação dos ex-alunos do curso de nutrição de uma universidade do interior paulista. Investigação. 2010; 10(2-3):54-9.

24. Viana EMN, Almeida PC, Soares NT, Luna MFG, Esmeraldo GROV. Adequação dos profissionais de saúde ao Programa de Saúde da Família: propostas e conquistas. Rev APS. 2010; 13(1):109-17.
25. Campos FCC, Faria HP, Santos MA. Planejamento e avaliação das ações em saúde. $2^{a}$ ed. Belo Horizonte: Coopmed; 2010.

26. Dimenstein $M$, Sever $A K$, Brito $M$, Pimenta $A L$, Medeiro V, Bezerra E. O apoio matricial em unidades de saúde da família: experimentando inovações em saúde mental. Saúde Soc. 2009; 18(1):63-74.

27. Feuerwerker LCM. Estratégias para a mudança da formação dos profissionais de saúde. Cad Ensino Currículo. 2001; 3(4):11-23.

28. Santos LAS. Educação alimentar e nutricional no contexto da promoção de práticas alimentares saudáveis. Rev Nutr. 2005; 18(5):681-92. http://dx. doi.org/10.1590/S1415-52732005000500011

29. Padua JG, Boog MCF. Avaliação da inserção do nutricionista na Rede Básica de Saúde dos municípios da Região Metropolitana de Campinas. Rev Nutr. 2006; 19(4):413-24. http://dx.doi.org/ 10.1590/S1415-52732006000400001

Recebido: novembro 21, 2013

Versão final: novembro 14, 2014 Aprovado: dezembro 17, 2014 